\title{
PSTM/NSOM Modeling by 2-D Quadridirectional Eigenmode Expansion
}

\author{
Manfred Hammer and Remco Stoffer
}

\begin{abstract}
A two-dimensional (2-D) model for photon-scanning tunneling microscopy (PSTM) of integrated optical devices is evaluated. The simulations refer to a setup where the optical field in the vicinity of the sample is probed by detecting the optical power that is transferred via evanescent or radiative coupling to the tapered tip of an optical fiber close to the sample surface. Scanning the tip across the surface leads to a map of the local optical field in the sample. As a step beyond the mere analysis of the sample device, simulations are considered that include the sample as well as the probe tip. An efficient semianalytical simulation technique based on quadridirectional eigenmode expansions is applied. Results for a series of configurations, where slab waveguides with different types of corrugations serve as samples, allow assessment of the relation between the PSTM signal and the local field distribution in the sample. A reasonable qualitative agreement was observed between these computations and a previous experimental PSTM investigation of a waveguide Bragg grating.
\end{abstract}

Index Terms-Integrated optics, near-field scanning optical microscopy (NSOM), numerical modeling, photon-scanning tunneling microscopy (PSTM), photonics.

\section{INTRODUCTION}

$\mathbf{P}$ HOTON-SCANNING tunneling microscopy (PSTM) or near-field scanning optical microscopy (NSOM or SNOM) becomes increasingly popular as a tool to study the local optical electromagnetic field close to the surface of devices from integrated optics/photonics. A typical setup (cf., e.g., [1]) consists of a tapered tip of an optical fiber, the probe, that is attached to the tuning fork of an atomic-force microscope (AFM). By scanning the probe at a narrow distance, typically a few tens of nanometers, across the sample surface, one generates simultaneously a topographical and an optical signal. While in general the optical part of the microscope can be operated in different schemes concerning illumination and detection, here we are interested in setups where the light is fed into the waveguiding sample structure by means of conventional coupling techniques of integrated optics and where the power detected at the end of the fiber that is attached to the probe tip is recorded as the optical signal.

Examples for these kinds of experiments include the mapping of fields in (multimode) dielectric optical waveguides [2]-[6], in an optical Y-junction [7], or in a Mach-Zehnder interferometer [1]. Other studies considered the evolution of optical fields behind a slit distortion in a waveguide [8], in waveguide Bragg gratings [9], in photonic-crystal slabs [10],

Manuscript received August 23, 2004; revised December 17, 2004.

The authors are with the MESA ${ }^{+}$Research Institute, University of Twente, 7500 AE Enschede, The Netherlands.

Digital Object Identifier 10.1109/JLT.2005.844498 or in photonic-crystal structures with defect cavities [11], [12]. PSTM observations of resonances in cylindrical integrated optical microresonators are reported in [13]-[15].

Besides the actual experiments, simplified microscope models have also been studied by means of numerical simulations, though mostly for different schemes of operation. The optical fields in the surrounding of dielectric (coated or uncoated) or metallic probe tips in two spatial dimensions are investigated in [16], [17] ([18] for related experiments), [19], and [20]. The simulations include also the interaction of the tip with simple objects on glass substrates: circular dielectric or metallic, nanometer-sized two-dimensional (2-D) particles (illumination via the probe), or dipole sources (single molecule fluorescence). Examples for studies in three spatial dimensions are [21] and [22], where the optical field around isolated, metallic, or coated dielectric circular-conical probe tips is considered or the simulations of the interaction between a conical uncoated dielectric tip with a freestanding glass bar of nanometer dimensions in [23]. A variety of computational techniques were applied, including the multiple multipole (MMP) method [24], calculations based on a Green's tensor description [25], [26], or the popular finite-difference time-domain (FDTD) simulations [27], [28].

All previously mentioned numerical investigations focus on the optical field inside and in the immediate vicinity of the probe tip and the simple, nanometer-sized samples. In particular, the proper modeling of particular probe shapes is aimed at, with the intention to assess the possibility of sub-wavelength resolution of the microscope, or with the intention to optimize the probe geometry. In view of the experimental attempts to map the optical fields in the considerably larger photonic structures, however, one would wish to include the entire sample device into the simulation, in order to examine the interaction of the probe tip with the (nontrivial) optical field that establishes in the device under test. An example is the large-scale, full three-dimensional (3-D) FDTD study of [29], where the PSTM observation of optical fields and band structure of a photonic-crystal slab has been simulated. Unfortunately, due to the computational complexity of the problem, the 3-D calculations [23], [29] remain restricted to a rather limited computational domain around the actual interaction region and to a rather limited number of probe/sample configurations.

Hence, for the present contribution, we looked at a highly simplifying 2-D model of the optical part of the PSTM microscope. Corrugated slab waveguides (cores with slits, short Bragg gratings) serve as samples. A half infinite piece of waveguide, oriented perpendicularly to the axis of the sample structures, represents the probe tip. This model is certainly limited with 

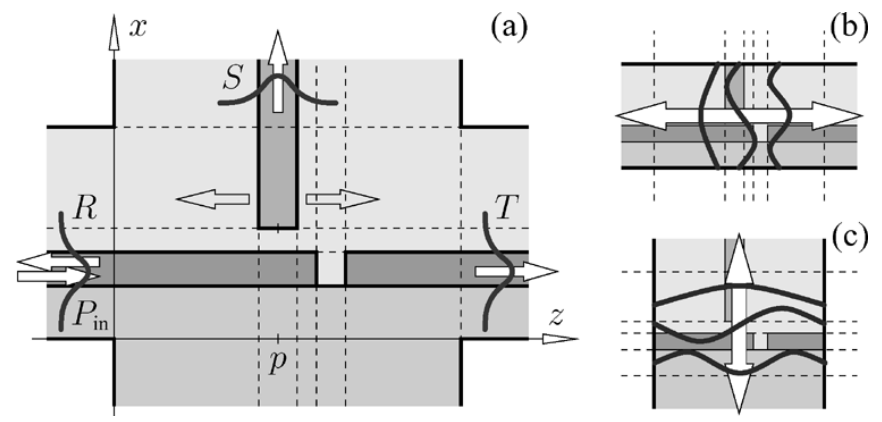

Fig. 1. (a): Schematic of the 2-D PSTM model, here consisting of a slab waveguide with a hole in the core, the sample, and a half-infinite vertical waveguide segment (the probe). Cartesian coordinates $x$ and $z$ denote the horizontal and vertical directions. $p$ is the $z$ position of the probe tip center. The dashed lines indicate the division of the structure into layers and slices, as required for the QUEP simulations. (b) and (c): QUEP basis fields; the solution of the scattering problem is expanded into eigenmodes of local one-dimensional (1-D) multilayer slab profiles that travel along the (b) horizontal and (c) vertical.

respect to the range of structures where it can be applied reasonably, and with respect to the aspects of the microscopy technique that can be captured. Nevertheless, efficient 2-D simulation techniques permit the inclusion of comparably large sample devices into the calculations and offer the possibility for detailed studies of the interaction between probe and sample. A recently proposed semianalytical method (quadridirectional eigenmode propagation, or QUEP) [30] constitutes a convenient tool for virtual experiments with this model.

After the introduction of the PSTM model, Section II-A gives a brief outline of the simulation technique. In Section III, a series of results for different sample structures are discussed. The examples allow estimation of how the signal detected via the fiber is related to the field intensity at the probe tip and how the presence of the probe influences the field distribution within the sample. Section IV compares an experimental PSTM investigation [9] of a waveguide Bragg grating with the results of corresponding 2-D simulations.

\section{2-D PSTM MODEL}

Fig. 1(a) introduces the PSTM model. The guided mode of the horizontal slab illuminates the sample from the left with input power $P_{\text {in }}$; the power $S$ associated with the upwards traveling guided mode of the vertical probe slab represents the primary PSTM signal. A PSTM scan is modeled by evaluating $S$ as a function of the probe center position $p$. Besides $S$, the guided transmitted and reflected power fractions $T$ and $R$ are recorded to assess in how far the presence of the probe alters the global optical transmission state of the sample.

We restrict the discussion to lossless isotropic materials, and consequently to uncoated, purely dielectric probes. For simplicity, we also assume that the probe moves along the sample at a constant $x$ level, i.e., that the probe does not follow the corrugation of the sample surface. This assumption is reasonable, for example, for the experiments of [9] (Section IV), where the probe tip diameter is larger than the topological features of the sample.

\section{A. Simulations by Quadridirectional Eigenmode Propagation}

The previous model assumptions lead to 2-D scattering (Helmholtz) problems with guided influx and outflux, for a purely real, isotropic, and piecewise constant permittivity profile, where all dielectric interfaces are parallel to one of the coordinate axes. The propagation of light with fixed frequency, given by the vacuum wavelength $\lambda$ is to be analyzed. Two classes of solutions with definite polarization exist: for transverse-electric (TE) waves, only the electric field component $E_{y}$ perpendicular to the $x-z$ plane is present ("perpendicular polarization"). In the transverse-magnetic (TM) case, the electric field is polarized in the $x-z$ plane ("parallel polarization"); then, usually the perpendicular magnetic component $H_{y}$ is used for a scalar description of the optical field. Note that within the 2-D model, these decoupled scalar field representations are exact.

The simulation technique as described in detail in [30] requires to enclose the interesting region in a rectangular window. A solution of the Helmholtz problem is then sought on a cross-shaped computational domain that consists of this interior window, together with the outwards unbounded external stripes that are connected to the four edges. On the four corner points of the inner rectangle and on the boundaries of the external regions, the basic field components $\left(E_{y}\right.$ for TE and $H_{y}$ for TM polarization) are assumed to vanish. The bold corners in Fig. 1(a) indicate the restriction of the computational domain.

To define the basis fields for the eigenmode expansion, two types of divisions of the computational domain are considered. On the one hand, one views the structure as a sequence of vertical slices [Fig. 1(b)], such that within each slice the refractive-index profile is constant along the $z$ axis. The modes associated with these individual slices ( $x$-dependent profiles, propagation along the $z$ axis) constitute one set of basis fields. On the other hand, the structure is decomposed into a stack of horizontal layers Fig. 1(c), where the permittivity is constant along the $x$ axis within each layer. The modes associated with the separate layers ( $z$-dependent profiles, propagation along the $x$ axis) form a second set of basis fields. In both cases, the mode spectra are discretized by artificial Dirichlet boundary conditions imposed on the edges of the inner rectangle of the computational domain. Bidirectional versions of propagating and evanescent modes up to certain orders $M_{x}$ (related to the division into slices) and $M_{z}$ (related to the layer decomposition) are taken into account. Modes traveling in the positive and negative $x$ and $z$ directions contribute; hence, one could call this a "quadridirectional" eigenmode expansion.

Superpositions of all basis modes establish the ansatz for the optical field, defined piecewise for the individual slices respectively layers. This ansatz satisfies the relevant wave equation everywhere in the computational domain, with the exception of the horizontal and vertical segment boundaries, where the Maxwell equations require certain continuity conditions to be satisfied. Consistent projection of these equalities onto the basis elements ("overlap" computations) with respect to suitable mode products [30], [31] allows extraction of a linear system of equations in the as-of-yet unknown coefficients of the eigenmode expansion. Assuming that the analytic basis modes are computed exactly, the emerging system of equations permits a stepwise solution. As far as only the segments inside the inner rectangle are concerned, the contributions of the horizontally and vertically propagating modes are decoupled. Conventional bidirectional 
eigenmode propagation (BEP) schemes [32], [33] can be applied; the present approach is related to the formulation given in [34] and [35]. The partial solutions [viewpoints Fig. 1(b) and (c)] are subsequently connected by the equations that belong to the continuity conditions at the four outer edges of the computational rectangle. Only this last (essential) combination step establishes a solution of the wave equation on the entire cross-shaped computational domain.

The optical influx is specified by prescribing the amplitudes of all inwards traveling basis fields on the exterior regions; in the examples of Section III typically only one amplitude of the incident guided mode of the horizontal slab is nonzero. This forms a right-hand side to the linear system, which is then solved for all remaining expansion coefficients, with the amplitudes of the outgoing basis fields on the exterior stripes as primary unknowns. In Section III, the optical powers $S, R$, and $T$ of the signal, reflected, and transmitted waves can be accessed directly by inspecting the squared coefficients of the outgoing normalized fundamental guided modes related to the upper, left, and right exterior regions.

The QUEP simulations treat the light propagation along both coordinate axes precisely alike. As an alternative to the viewpoint of the cross-shaped computational domain, the perpendicular, dependent superposition of BEP expansions may be viewed as a way to establish fully transparent boundary conditions (an exception is the corner points) for the inner rectangular computational window, with a straightforward possibility of modeling guided wave influx and outflux. Though somewhat restrictive what concerns the geometrical variability, the technique offers an accurate and quite efficient platform for computational studies of the PSTM model.

\section{NUMERICAL RESUlTS}

Given a guided wave input into the sample of power $P_{\text {in }}$, the squared amplitude of the normalized upwards guided mode of the probe represents the signal $S$ in our model (usually the major recorded quantity in an actual PSTM experiment; note that for a vertically homogeneous lossless fiber, the power $S$ detected at the upper end of the probe does not depend on the length of the waveguide). We observed a predominant expectation that $S$ should be a measure of the local intensity, i.e., a quantity $\sim\left|E_{y}\right|^{2}(\mathrm{TE})$ or $\sim\left|H_{y}\right|^{2}(\mathrm{TM})$, at the end of the probe tip. Therefore, the plots in the following sections compare curves for $S / P_{\text {in }}$ and data for $\left|E_{y}\right|^{2}(g, p)$ or $\left|H_{y}\right|^{2}(g, p)$, respectively, where $g$ and $p$ are the $x$ and $z$ coordinates of the center of the tip facet. The presence of the probe may change the local intensity, hence two curves for that quantity are given: one set of data points represent a scan along the line $x=g$ across a solution of the scattering problem without the probe, i.e., the field in the unperturbed sample (replace the $p$ axis in the plots by the $z$ coordinate). A second curve corresponds to the local intensity in a configuration where the probe tip center is situated at the observation point. Identifiers $s$ (sample only) and $s+p$ (sample and probe) distinguish these values.

In order to facilitate a quantitative comparison, for each figure the curves $s$ and $s+p$ for the local intensities have been scaled by a common factor such that the $s$ level coincides with the signal data $S$ at a single position. Circle symbols mark these scaling

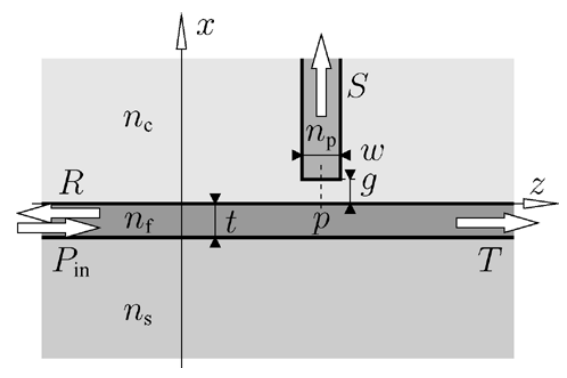

Fig. 2. PSTM model configuration. Sample: a slab waveguide, thickness $t=0.2 \mu \mathrm{m}$, refractive indexes $n_{s}=1.45$ (substrate), $n_{f}=2.0$ (film), and $n_{c}=1.0$ (cladding, background). Probe: a vertical slab, width $w=100 \mathrm{~nm}$, and refractive index $n_{p}=1.5$. Illumination by light with a vacuum wavelength of $\lambda=0.633 \mu \mathrm{m}$. QUEP parameters: computational domain $(x, z) \in[-3.0,3.0] \times[-3.0,3.0] \mu \mathrm{m}^{2}$, and number of expansion terms $M_{x}=M_{z}=80$.

points in the corresponding plots. The scaling represents the common procedure to "match" measurements of relative quantities; here, the proportionality of the local field $s$ and the signal power $S$ is to be investigated. Naturally, the choice of the scaling points influences to a certain degree the impression given by the plots. We have selected these positions such that the local proportionality between the $S$ and $s$ curves becomes apparent; none of the conclusions depends on these choices.

All QUEP results shown hereafter have (roughly) been checked for convergence with respect to the extension of the inner computational window and with respect to the density of the spectral field discretization. Still, for completeness, the corresponding data for the computational intervals and for the numbers of basis modes per propagation direction and per slice $M_{x}$ or per layer $M_{z}$ are given in the captions along with the structural parameters of the computations. Note that the transparent boundary conditions implemented by the QUEP algorithm permit outgoing guided and nonguided waves to leave the inner computational rectangle across the four edges. For the final solution, Dirichlet boundary conditions apply only on the corner points, and on the boundaries of the exterior slices, as indicated in Fig. 1(a).

\section{A. Probing Evanescent Fields}

An ideal uncorrugated slab waveguide serves as the first sample. Fig. 2 introduces the trial structure. With the exponential decay of the guided slab mode profiles, here the unperturbed fields in the vicinity of the sample structure are known exactly. Hence, the slab permits a most straightforward comparison of the PSTM signal with the probed optical field.

For the $z$-invariant sample, the power levels observed for the signal $S$, transmission $T$, and reflection $R$ during a horizontal scan at constant height $g$ should obviously not depend on the probe position $p$. This can be used for a consistency check of the simulations: due to the particularities of the spectral discretization, one must expect a dependence of the results on the position where the vertical slab is placed inside the computational domain. Reassuringly, for positions where the guided mode profile associated with the probe is contained reasonably within the computational window, we found hardly any variation of $S, T$, and $R$ with $p$ on a scale that becomes relevant for the subsequent configurations with comparable simulation parameters. 

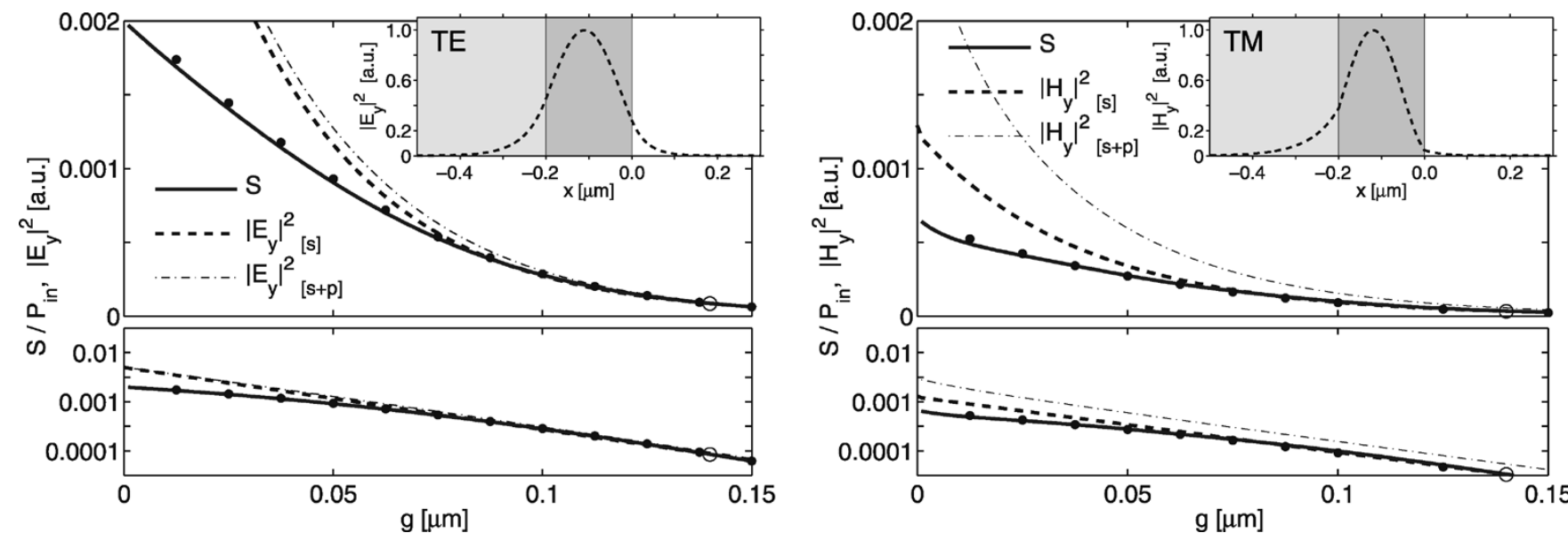

Fig. 3. Vertical scan over the slab of Fig. 2, for TE- (left) and TM-polarized illumination (right). The curves show the dependence of the PSTM signal $S$ (continuous) and the local field intensities at the probe tip center $\left|E_{y}\right|^{2}$ or $\left|H_{y}\right|^{2}$ without the probe ( $s$, dashed) and with the probe ( $s+p$, dashed-dotted), on the width $g$ of the gap between probe and sample. FDTD results for the signal (cf. Section III-A-1) are indicated by filled circular markers. Insets: Intensity profiles of the fundamental modes supported by the sample.

For the present parameters, the relative signal levels for TE polarization are by about a factor 4 larger than those for TM waves. A possible explanation could view the perturbation effected by the probe as a dipole source at the probe facet. Its directional radiation pattern includes the probe axis in case the dipole is oriented along the $y$ axis, i.e., for TE polarization. For TM-polarized light, the dipole is oriented in the $x-z$ plane more or less along the probe axis, such that the probe receives much less optical power. We therefore restrict most of the following simulations to TE-polarized illumination, although, for the present high-contrast structures, one must expect pronounced differences between the polarizations.

A vertical scan turns out to be more interesting. According to corresponding claims based on experimental observations, e.g., in [2], [4], [5], [10], and [36], the signal should be proportional to the "intensity at the end of the probe," here interpreted as the square of the local electromagnetic field that exists at the position of the center of the probe facet, for an absent probe. Hence, if the probe is lifted upwards away from the sample, one expects a purely exponential decay of the signal power with the gap distance $g$, according to the exponential shape of the guided mode profile supported by the sample. Fig. 3 shows the results of our corresponding calculations.

Contrary to the expectation, here we cannot confirm a strict proportionality between the signal and the field intensity at the probe tip. While the squares of the exponential tails of the sample mode profiles are represented by exact straight lines in the logarithmic lower plots of Fig. 3, the lines related to the PSTM signal are curved. This holds also for relatively large probe-sample separations, although, according to the plots, for large $g$ an exponential fit seems to be rather evident.

Most PSTM experiments, however, are operated with a gap between sample and probe of about $10 \mathrm{~nm}$. In that regime, the proportionality between the signal and the local field intensity in the unperturbed sample is pronouncedly violated. These findings are actually in accordance with an early measurement reported in [2], where a planar silicon-oxide/silicon-nitride waveguide of thickness $160 \mathrm{~nm}$ is considered, illuminated by TE-polarized light at a wavelength of $632.8 \mathrm{~nm}$, i.e., a configuration quite similar to Fig. 2. Also there a systematic deviation of the PSTM signal from the straight line in the logarithmic plots can be observed, most apparent for small probe-sample separations.

Interestingly, the influence of the probe on the field strength at its tip turns out to be much less pronounced. Fig. 3 shows hardly any change between the $s$ and $s+p$ data for TE polarization and a change by merely an almost constant factor for TM polarized waves. On the scale of the figure, the curves for $\left|E_{y}\right|_{s+p}^{2}$ or $\left|H_{y}\right|_{s+p}^{2}$ retain the exponential shape of the unperturbed sample field $\left|E_{y}\right|_{s}^{2}$ or $\left|H_{y}\right|_{s}^{2}$, respectively, deviating from the nonexponential behavior of $S(p)$.

In Fig. 4, we try to visualize what happens when the probe enters the evanescent field around the sample. Without the probe, only the plane-wave fronts of the guided slab modes are visible. If the probe is introduced, as a large-scale effect waves of cylindrical-like shapes appear, originating from the probe tip, with different material-dependent wavelengths in the substrate and cover regions. While the angular distribution is almost symmetrical for TE polarization, the TM waves exhibit a more directional forward-oriented wave pattern. Also this difference could be explained by the dipole-source viewpoint mentioned previously.

Local field distortions due to the probe are revealed best by the curvature of the contours in the plots of the absolute field values. Without the probe, the contours are straight horizontal lines, spaced according to the mode profile of the $z$-homogeneous slab. Apparently some disturbance is necessary to force optical power up the fiber probe. Note that here all effects of the probe relate to rather small fractions of the total incident optical power: for the gap $g=10 \mathrm{~nm}$, the guided relative transmission and reflection evaluate to $T=0.994, R=7 \cdot 10^{-5}$ (TE), and $T=0.990, R=1 \cdot 10^{-5}(\mathrm{TM})$; only the remaining parts of the power budgets are distributed between the signal and the radiation.

1) Comparison With FDTD Results: In order to exclude possible artefacts of the simulation technique, and as an additional check of the somewhat unexpected nonproportionality of the signal and the local field intensity, we verified the above findings by means of an entirely independent numerical 

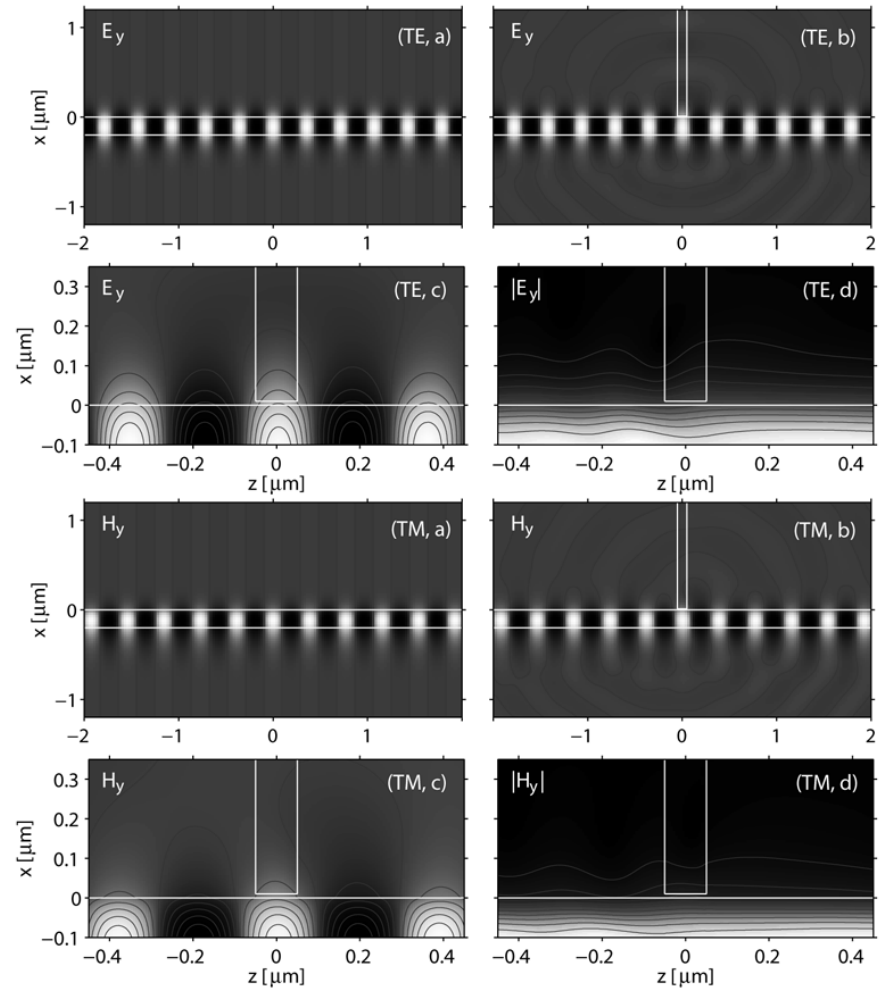

Fig. 4. Optical field around the slab waveguide of Fig. 2, for TE- (top) and TM-polarized light (bottom). Gray-scale levels correspond (a)-(c) to the real stationary physical fields (snapshots at an arbitrary time) or (d) to the local absolute value of the principal component. (a) Separate sample. (b): Sample and probe; the contours indicate the zero level. (c) and (d): Magnification of the region around the probe tip (c) 11 or (d) 10 equidistant contour lines. The probe-sample distance is $g=10 \mathrm{~nm}$.

method. A rigorous 2-D FDTD scheme [27], [28], [37] has been applied; below we relate briefly the details of these calculations.

A computational window of $4.2 \times 4.2 \mu \mathrm{m}^{2}$ is discretized uniformly by a mesh with step sizes of $0.0125 \mu \mathrm{m}$. Perfectly matched layer (PML) boundary conditions enclose the computational domain, with a width of $0.4 \mu \mathrm{m}$, a quadratic envelope, and a strength such that the theoretical reflectivity of a wave propagating through the background material at normal incidence is $10^{-6}$. The interior of the computational window contains the centered sample waveguide and the probe tip with parameters as given for Fig. 2.

Properly polarized modal fields are launched into the sample core using the total field/scattered field approach [28]. Their amplitude is raised according to a half-Gaussian curve with a waist of $10 \mathrm{fs}$, with the maximum being reached at $40 \mathrm{fs}$. After this time, the incident field amplitude is kept constant. The simulation runs for a time of $102.4 \mathrm{fs}$ with a time step of $0.025 \mathrm{fs}$, until a stationary field is established, after which the overlap with the mode of the probe tip at a distance of $0.5 \mu \mathrm{m}$ from the top of the calculation window is evaluated. Fig. 3 exhibits an excellent agreement between the FDTD and the QUEP results.

What concerns the computational effort, the present QUEP implementation completes the solution of a single scattering problem (one $g$ value in Fig. 3 in about $6 \mathrm{~s}$ on an up-to-date PC, using about $4 \mathrm{MB}$ of memory, while our 2-D FDTD program takes about $67 \mathrm{~s}$ and occupies about $6 \mathrm{MB}$ of memory. Note, however, that the QUEP approach addresses directly the

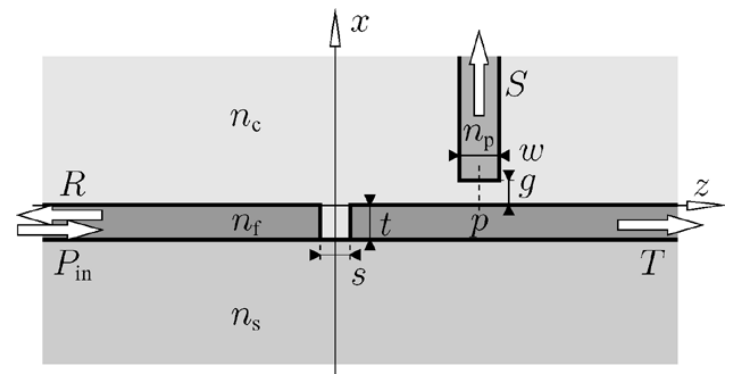

Fig. 5. PSTM model configuration. Sample: Slab waveguide with a hole in the core, width $s=0.2 \mu \mathrm{m}$. Other sample and probe parameters are as in Fig. 2, with a constant probe-sample distance $g=10 \mathrm{~nm}$. Only TE-polarized illumination with a wavelength of $\lambda=0.633 \mu \mathrm{m}$ is considered. QUEP parameters: $(x, z) \in[-3.0,3.0] \times[-3.1,3.1] \mu \mathrm{m}^{2}, M_{x}=M_{z}=80$.

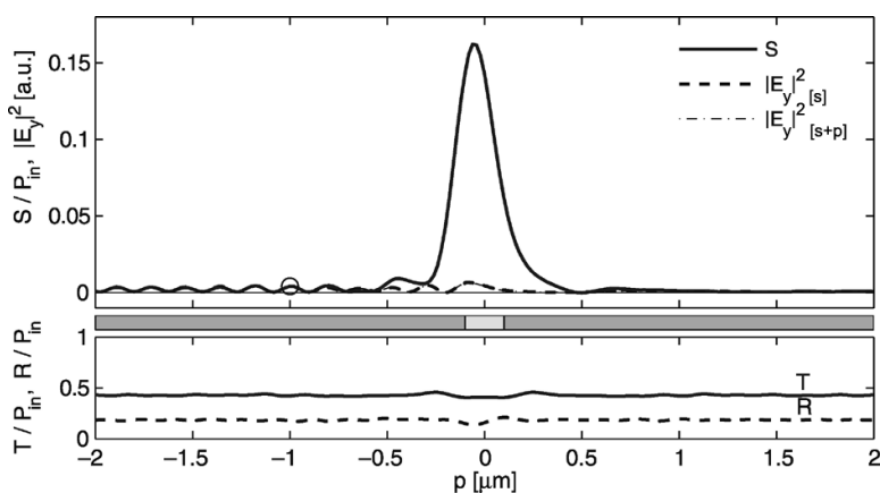

Fig. 6. Horizontal PSTM scan across the waveguide defect of Fig. 5, signal $S$, and local field intensity $\left|E_{y}\right|^{2}$ (top) versus the $z$ position $p$ of the probe center. Bottom: Guided power transmission $T$ (continuous) and reflection $R$ (dashed).
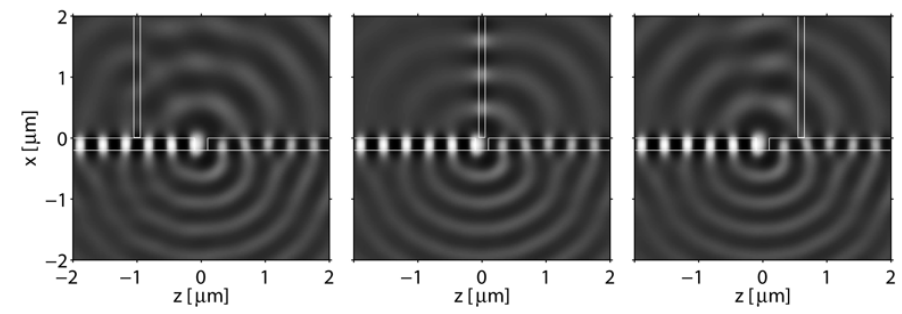

Fig. 7. Optical field around the hole in the waveguide of Fig. 5, for probes at positions $p=-1.0,0.0,0.6 \mu \mathrm{m}$. The gray scales correspond to time snapshots of the stationary physical electric field $E_{y}$ of the TE waves.

frequency-domain (Helmholtz ) problem that is of interest here and does not - as is the case with the FDTD — compute an entire time evolution of an optical signal, that is afterwards discarded.

\section{B. Hole Defect in a Slab Waveguide}

As an example for a most simple, localized corrugation, a square-hole defect is introduced into the former waveguide sample. Fig. 5 specifies the geometry and the relevant parameters. After the basic characterization of the model "tool," from now on the simulations will be restricted to TE-polarized illumination, and to scans with a constant height $g$. Fig. 6 summarizes the results of a PSTM scan across the hole distortion; Fig. 7 shows three examples for the resulting optical field.

Without the probe, the hole reflects $R=19 \%$ and transmits $T=43 \%$ of the incident power; the remaining $38 \%$ are lost to nonguided, radiated fields. The superposition of the unit 


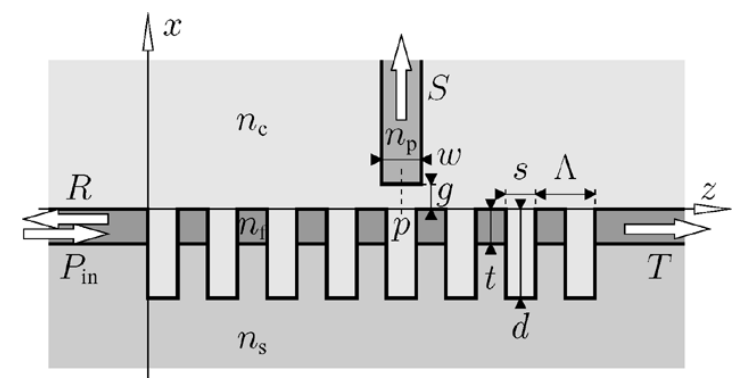

Fig. 8. PSTM model configuration. Sample: Short Bragg grating, eight slits of width $s=0.11 \mu \mathrm{m}$, period $\Lambda=0.21 \mu \mathrm{m}$, and etching depth $d=0.6 \mu \mathrm{m}$. Other sample, probe, and scan parameters are as given for Figs. 2 and 5. QUEP parameters: $(x, z) \in[-3.0,3.0] \times[-3.0,4.58] \mu \mathrm{m}^{2}, M_{x}=100$, and $M_{z}=$ 120.

input and the guided reflection forms partly standing and traveling waves in the input core segment; the confined transmission leads to outwards traveling waves in the ongoing core segment. Both the reflected and transmitted parts of the confined fields undergo a transient oscillation until the lateral shapes of the waves are adapted to the particular profile of the guided mode further away from the defect. The hole acts as a strong localized source, where the radiation consists of waves with cylindrical shapes that originate from the hole, with wavelengths according to the different refractive indexes in the substrate and the cover regions.

If the probe is moved along the sample in a region sufficiently far away $(|p| \geq 0.7 \mu \mathrm{m})$ from the hole, the PSTM signal follows nicely the local intensity of the predominantly evanescent waves that are present in those regions close to the sample surface. The almost periodic intensity pattern of the standing waves can be observed in the input segment. Beyond the hole, the signal corresponds to the lower constant level of the outgoing guided wave, with a shallow modulation due to the transient adaption to the precise mode profile. The radiated waves from the hole that reach the probe in these regions are partly reflected and transmitted by the vertical slab (cf. the third plot of Fig. 7) but do not significantly couple to the upwards traveling mode of the probe, i.e., do not contribute to the signal.

Close to the hole, however, the signal exhibits a strong peak, which obviously must be attributed to direct scattering from the hole upwards into the probe tip. Here, the relation between the local field and the signal is entirely lost. With a full-width at half-maximum of $0.23 \mu \mathrm{m}$, the peak is only moderately wider than the defect; the peak maximum is located $50 \mathrm{~nm}$ to the left of the center of the hole. If the probe is positioned directly above the defect, it collects most of the waves that are otherwise radiated into the upper half space (cf. the middle inset of Fig. 7).

\section{Short Waveguide Bragg Grating}

A periodic sequence of holes in a waveguide core forms a waveguide Bragg grating, or "1-D photonic crystal." Fig. 8 introduces a short, deeply etched high-contrast device that shall serve as the next sample. Figs. 9 and 10 collect the corresponding results of the PSTM model.

The geometry of the grating has been adjusted such that it acts as a strong reflector at the given wavelength. Without the

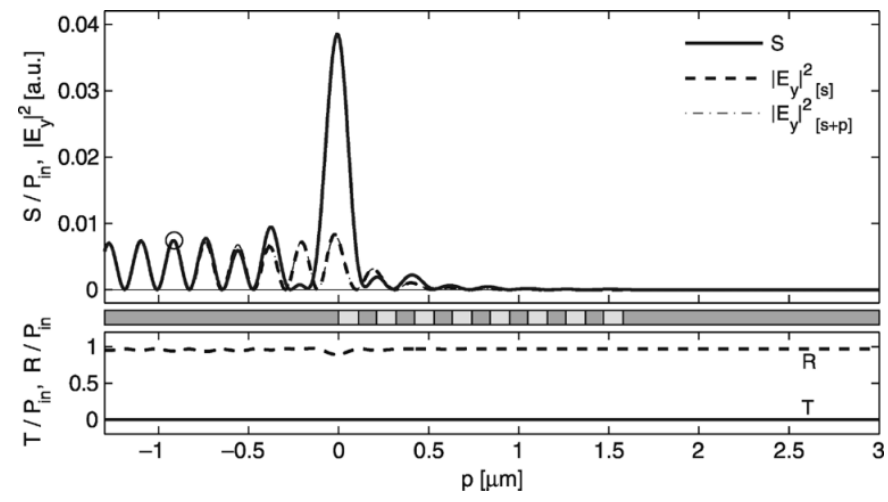

Fig. 9. Scan along the Bragg grating of Fig. 8; signal $S$, local field intensity $\left|E_{y}\right|^{2}$, and transmitted and reflected power $T$ and $R$ as a function of the probe position $p$.
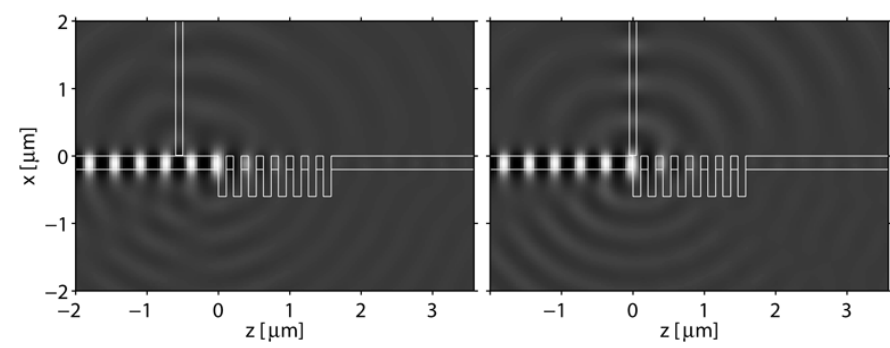

Fig. 10. Light propagation through the grating of Fig. 8; snapshots of the electric field $E_{y}$ for probe positions $p=-0.55 \mu \mathrm{m}$ and $p=0.0 \mu \mathrm{m}$.

probe, $R=97 \%$ of the input power is reflected, and about $T=$ $0.1 \%$ is transmitted to right-traveling guided modes. The low amount of radiation emanates mainly from the transition from the homogeneous core to the periodically corrugated segment at $z=0$; Fig. 10 shows the interference of these waves with the radiation caused by the probe tip in the substrate and cover regions.

The PSTM signal of Fig. 9 remains restricted to the region $p<1 \mu \mathrm{m}$ where optical power is actually present in the sample. For positions $p<-0.5 \mu \mathrm{m}$ before the transition to the grating, the field around the sample consists of interfering forward- and backward-propagating waves with the same mode profile shape and is scanned at constant height. Hence, the nonproportional effects (Section III-A) are not relevant here. Just as in Section III-B, the signal follows the local field intensity at the probe facet.

In the transition region around $p=0$, however, the signal and the sample field intensity deviate considerably. A pronounced peak appears, as before to be attributed to the direct scattering into the probe tip. Here, it is accompanied by "sidelobes," where the signal is actually much lower than the respective sample field intensity; the second but last maximum of the rather regular original standing-wave pattern is suppressed almost completely in the signal. Interference effects can obviously also lead to a significant underestimation of the local intensity in the PSTM measurement.

The signal level of 0.04 found at the peak maximum is actually larger than the total amount of power that is lost to radiation if the probe is absent. Apparently, when positioned close to the first discontinuity of the grating the probe does not only collect 


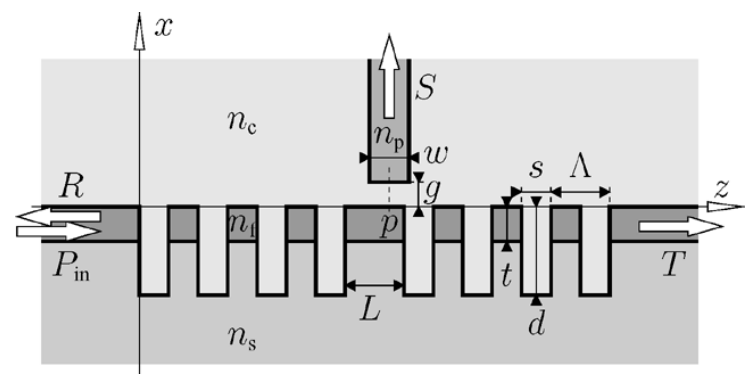

Fig. 11. PSTM model configuration. Sample: Short Bragg grating with a central defect, length $L=0.2275 \mu \mathrm{m}$. Other sample, probe, and scan parameters are as given for Figs. 2, 5, and 8. QUEP parameters: $(x, z) \in[-3.0,3.0] \times[-3.0,4.7075] \mu \mathrm{m}^{2}, M_{x}=100$, and $M_{z}=120$.

most of the optical power that is radiated upwards, but it actually raises the level of radiation. The curve $R(p)$ shows a corresponding dip close to $p=0$; there, the guided reflection drops to about $89 \%$. Similar changes of the transmission properties of the sample (a rather moderate on the scale of Figs. 6 and 9) were also observed for the former hole defect. In the following section, we consider a structure where the probe interacts with the sample on a much stronger level.

\section{Resonant Defect Cavity}

Introduction of a localized distortion in an otherwise periodic grating can cause drastic changes in the spectral response [38]. Here, we realize the defect by enlarging the central "tooth" of the former waveguide Bragg grating. Fig. 11 summarizes the geometrical details; Figs. 12 and 13 illustrate what happens when the PSTM tip scans across the structure.

One may view the device as a sequence of two reflectors, each consisting of a Bragg grating with four grooves, that enclose a short homogeneous waveguide segment. The familiar Fabry-Pérot model can be applied to explain the occurrence of resonant states within the frequency bandgap of the original long Bragg grating. The homogeneous section acts as a narrow cavity; resonances manifest as localized transmission peaks in a frequency interval where the device otherwise reflects most of the optical power. The cavity length $L$ in the specification of Fig. 11 has been adjusted such that the defect grating supports a transmission resonance at the vacuum wavelength that will be applied during the PSTM scan.

In its unperturbed state, the structure transmits $T=56 \%$ and reflects $R=6 \%$ of the incident power. Radiation originates mainly from the two input and exit transitions between the grating segments and the external homogeneous waveguides, and from the central cavity. The resonance establishes a predominantly standing-wave pattern, with two alternating maxima and a central node in the cavity segment, and outwards quickly decaying amplitudes in the grating regions (see the dashed line for $\left|E_{y}\right|_{s}^{2}$ in Fig. 12).

The phenomena identified in the previous examples can be found again in the present PSTM scan: one observes the adequately resolved outer standing-wave pattern and the scattering peaks with sidelobes around the transitions to the outer homogeneous segments. Different from the examples in Sections III-B and III-C, drastic changes in the transmitted and reflected power

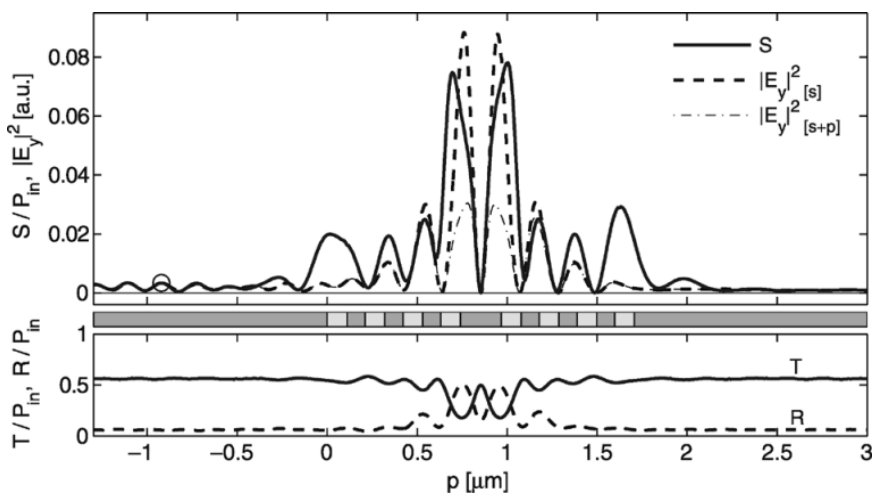

Fig. 12. PSTM scan across the defect grating of Fig. 11; signal $S$, local intensities $\left|E_{y}\right|^{2}$, and the transmission $T$ and reflection $R$ effected by the structure, versus the position $p$ of the probe.
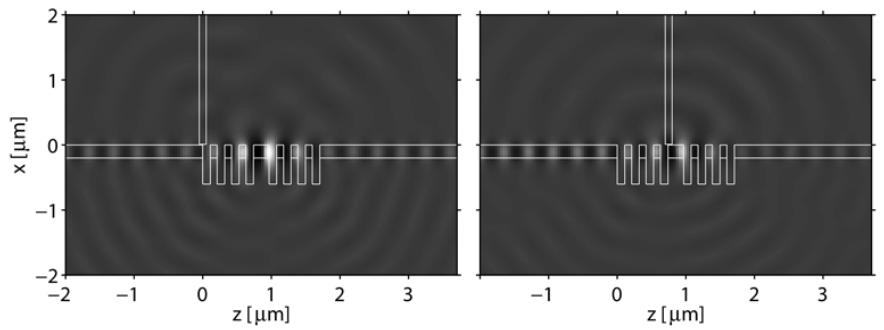

Fig. 13. Optical field snapshots for the defect cavity of Fig. 11, with the probe located at $p=0.0 \mu \mathrm{m}$ (first discontinuity, left) and at $p=0.75 \mu \mathrm{m}$ (in the defect region, right). The gray-scale levels of the plots are comparable.

levels appear, if the probe scans across the cavity region. The changes in $T$ and $R$ are accompanied by a pronounced difference between the $\left|E_{y}\right|_{s}^{2}$ and $\left|E_{y}\right|_{s+p}^{2}$ curves, i.e., between the field intensity at the position of the tip facet without and with the probe.

Obviously here the probe effects a major change of the global optical state in the sample, i.e., causes a breakdown of the resonance. Extremal levels of $T=0.18$ and $R=0.50$ are reached for $p=0.75 \mu \mathrm{m}$. The field in the sample experiences the strongest influence if the probe is positioned close to one of the two maxima of the unperturbed resonant field pattern. The raised reflection is clearly visible in the second inset of Fig. 13 as the larger strength of the standing-wave pattern. Somewhat surprisingly, the signal recorded for the cavity region is not too far off from the local intensity in the unperturbed sample. Apparently, the contribution of the scattering to the signal compensates for the decay of the local intensity due to the resonance breakdown.

The precisely opposite effect can be achieved in a structure with slightly modified cavity length $L=0.224 \mu \mathrm{m}$. For an absent probe, this specifies an off-resonance configuration at the operation wavelength, with a rather high reflection $R=0.70$ and lower transmission level $T=0.15$. During the scan along the defect, one observes again pronounced variations in $T$ and $R$, and in the local field intensity, now with the roles of $T$ and $R$ reversed when compared with Fig. 13. Around the position $p=0.75 \mu \mathrm{m}$, the extremal values of $R=0.30$ and $T=0.24$ are found. Here, the probe shifts the system toward a resonance that does not occur in the isolated sample device. 

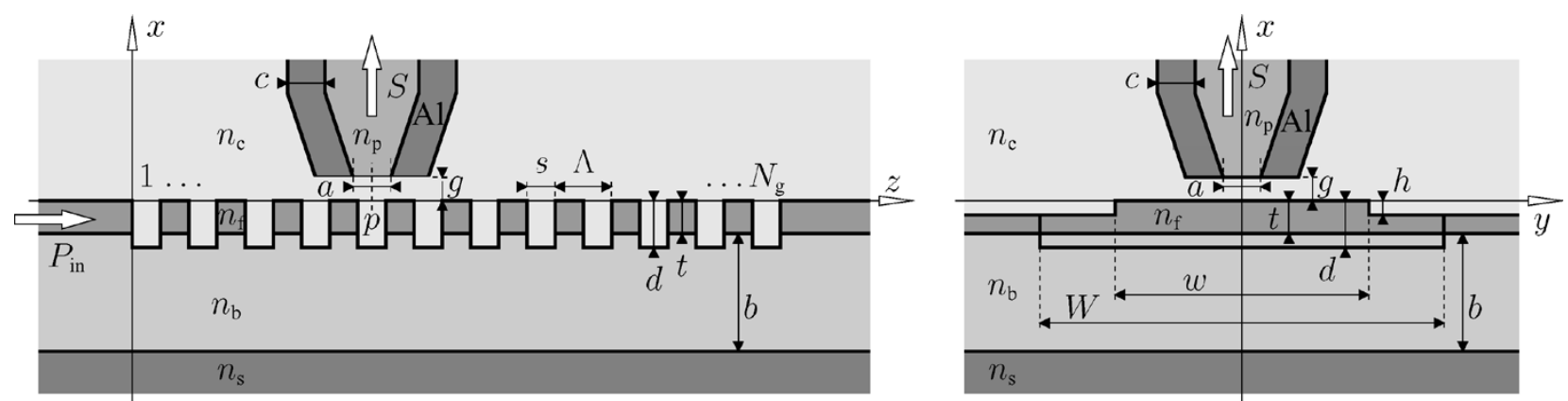

Fig. 14. Schematic of an experimental PSTM configuration [9], cross-section views of planes parallel (left) and perpendicular (right) to the waveguide axis. Sample: Rib waveguide with a series of rectangular slits, with refractive indexes $n_{s}=3.4$ (substrate), $n_{b}=1.45$ (buffer), $n_{f}=2.01$ (core), $n_{c}=1.0$ (cover), film thickness $t=55 \mathrm{~nm}$, rib etching depth $h=11 \mathrm{~nm}$, rib width $w=1.5 \mu \mathrm{m}$, transverse slit width $W=2.5 \mu \mathrm{m}$, buffer layer thickness $b=3.2 \mu \mathrm{m}$, grating period $\Lambda=220 \mathrm{~nm}$, longitudinal slit width $s=110 \mathrm{~nm}$, slit etching depth $d=70 \mathrm{~nm}$, and number of grooves $N_{g}=15$. Probe: Tapered cylindrical fiber tip with aluminum coating, aperture diameter $a \approx 80 \mathrm{~nm}$, cladding thickness $c \approx 100 \mathrm{~nm}$, core refractive index $n_{p}=1.5$, and distance probe/rib surface $g=10 \mathrm{~nm}$. The Bragg grating is illuminated by TE $(y-)$ polarized light with a vacuum wavelength $\lambda=0.6328 \mu \mathrm{m}$.

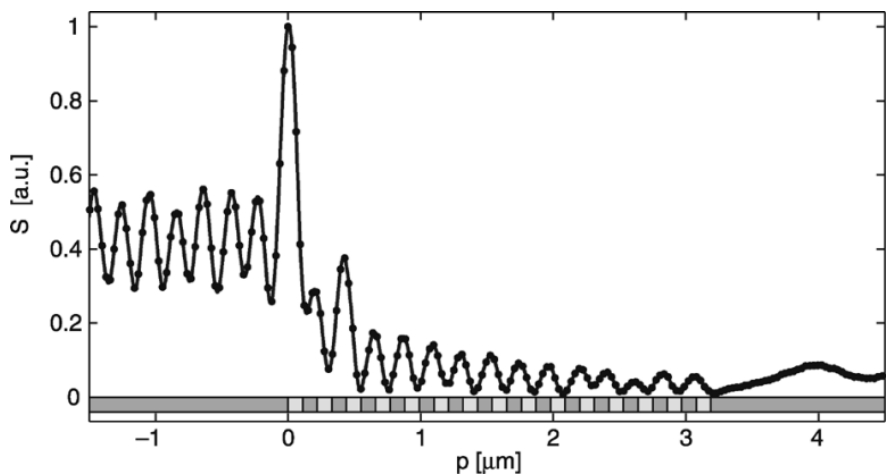

Fig. 15. PSTM scan along the center of the corrugated rib of Fig. 14; signal power $S$ depending on the $z$ position $p$ of the probe; experimental data from [9].

\section{BRAGG GRATING: EXPERIMENTAL RESULTS}

A waveguide Bragg grating is investigated in an actual experiment in [9]. The sample consists of a shallow rib waveguide with a $\mathrm{Si}_{3} \mathrm{~N}_{4}$ core, supported by a relatively thick $\mathrm{SiO}_{2}$ buffer layer on a silicon substrate. A series of wide, deeply etched rectangular slits forms the grating. Fig. 14 introduces schematically the experimental parameters that are relevant for the present discussion.

For the PSTM observation, the sample is illuminated via end-facet coupling through a microscope objective. By using the feedback from the AFM signal, the optical probe is raster-scanned at a constant distance across the surface of the rib waveguide. Since the diameter of the probe facet (including the coating) is larger than the slit width, at least for probe positions above the actual rib one can assume a constant scan height. One obtains a "top view" PSTM image of the optical field above the sample structure, together with the topological information that allows to precisely relate the optical data to the positions on the sample. Fig. 15 shows a section of the 2-D PSTM image along the axis of the sample waveguide. (See [9] for further details concerning the experimental setup and the full measurement results.)

\section{A. 2-D QUEP Model}

Due to the wide weak lateral features of the Bragg grating, one can expect that the optical field also varies only slowly along

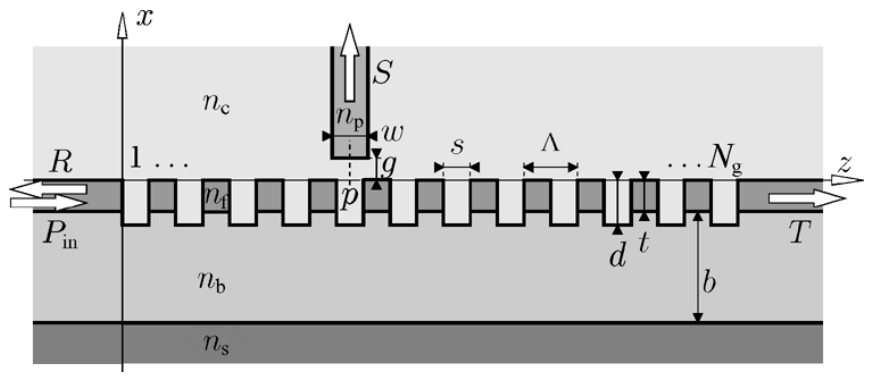

Fig. 16. Two-dimensional model of the 3-D configuration in Fig. 14. Parameters, sample: $n_{s}=3.4, n_{b}=1.45, n_{f}=2.01, n_{c}=1.0, t=55 \mathrm{~nm}$, $b=3.2 \mu \mathrm{m}, d=70 \mathrm{~nm}, \Lambda=220 \mathrm{~nm}, s=110 \mathrm{~nm}, N_{g}=15$; probe: $w=100 \mathrm{~nm}, n_{p}=1.5 ; g=10 \mathrm{~nm}, \mathrm{TE}, \lambda=0.6328 \mu \mathrm{m}$. QUEP parameters: $(x, z) \in[-3.5,1.5] \times[-2.0,5.2] \mu \mathrm{m}^{2}, M_{x}=80$, and $M_{z}=100$.

the $y$ direction, when compared with the longitudinal wavelength. We therefore take a cross section along the waveguide axis as the basis for the definition of the sample in our 2-D simulations. This leads to the model geometry of Fig. 16. While the buffer layer is of sufficient thickness to prevent leakage of the guided fields into the substrate, it cannot a priori be excluded that reflections of scattered waves from the substrate contribute to the PSTM signal. Therefore, the substrate/buffer layer interface is included in the simulations (although not the strong material attenuation of the silicon). Figs. 17 and 18 show the corresponding results of the model.

With the small film thickness, the waveguide operates under conditions very close to cutoff. As a consequence, a large fraction of the modal profile stretches out into the buffer region below $x=-70 \mathrm{~nm}$, where those fields do not encounter the slits directly. Therefore, the grating constitutes only a moderate reflector at the given wavelength. The 2-D simulations predict a guided transmission of $T=42 \%$ and a reflection of only $R=9 \%$. According to Fig. 18, when entering the corrugated region, the major part of the light waves forms of a strong beam that propagates at a shallow angle underneath the slits. Beyond the grating, a fraction of that power "leaks" back into the ongoing guided mode. Together with waves that are reflected from the buffer layer/substrate interface, the transient field adaption to the profile of the transmitted guided mode establishes a recovery pattern with a particular scheme of local minima (not 


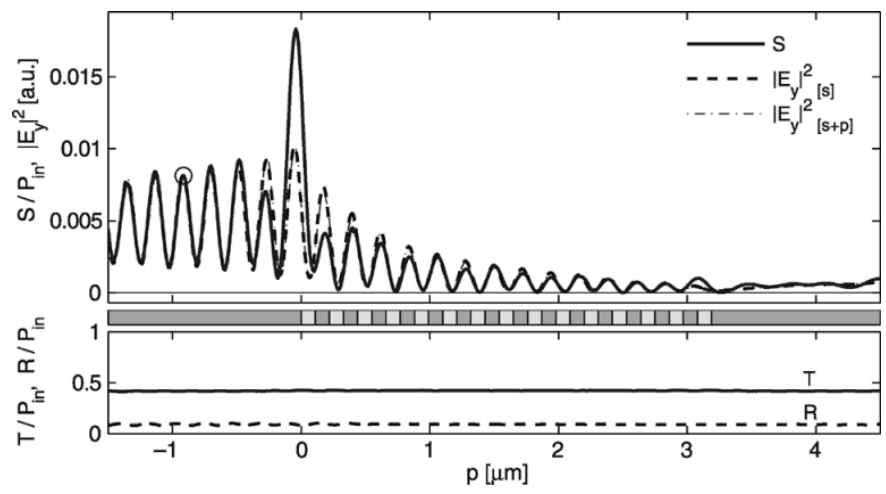

Fig. 17. PSTM scan along the waveguide Bragg grating of Fig. 16; 2-D model results for Fig. 15. Signal $S$, local field intensities $\left|E_{y}\right|^{2}$, and guided transmission $T$ and reflection $R$ versus the probe position $p$.

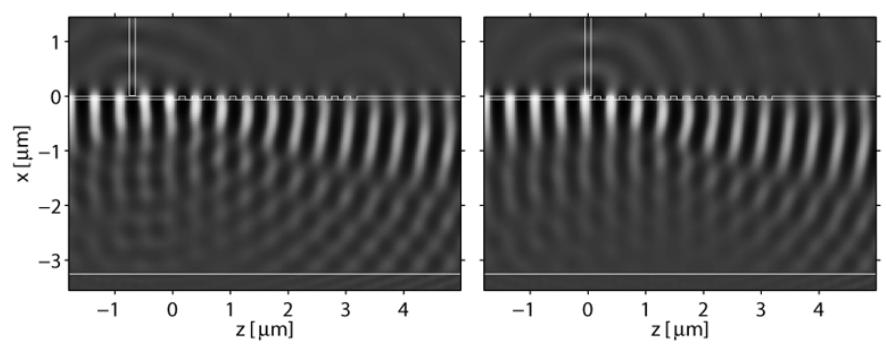

Fig. 18. Snapshots of the optical electric field according to the 2-D Bragg grating model of Fig. 16, for probe positions $p=-0.7 \mu \mathrm{m}$ (left) and $p=$ $0.0 \mu \mathrm{m}$ (right, beginning of the grating).

shown in Fig. 18). Details of the experimental observation of this pattern are discussed in [9].

For the separate sample structure, Fig. 17 indicates that the field intensity $\left|E_{y}\right|_{s}^{2}$ above the slits drops from the initially large level at the entrance to the grating to quite small values at the exit, where the exit value is much lower than the level that corresponds to the large overall transmission. The decay, and the subsequent recovery of the local intensity, can also be explained by the gradual displacement of the beam center toward the substrate, and by the back-flow of the waves into the core afterwards.

We find a good qualitative agreement of the simulations in Fig. 17 with the experimental data of Fig. 15. In the experiment as well as in the model, the PSTM signals map adequately the standing-wave pattern in front of the sample, the decay in the grating region, and the recovery process after the end of the grating. Since these features are indeed present in the $\left|E_{y}\right|_{s}^{2}$ curve of the model, one can expect that also the field intensity in the real device has a similar shape in the respective regions. Around the input transitions, however, the simulated PSTM signal does not follow the much more regular local field intensity. Since the large scattering peak and the partial suppression of the adjacent maxima occur in the experimental data as well, the model clearly identifies these features as artefacts of the PSTM observation (cf. the corresponding remarks in [9]).

Although the approximation of an only weak lateral confinement of the optical field in the 3-D sample is very plausible, the experimental probe has a circular aperture, which implies a strong localization of the upwards traveling signal waves. Also other experimental circumstances like the effects of the aluminum coating are not incorporated into the model; hence, one can hardly expect a better quantitative agreement between Fig. 15 and 17. Note, however, that the scattering peak as predicted here for the uncoated model probe appears in the experimental data as well. If one interprets the peak as a consequence of direct scattering into the probe aperture, the aluminum coating does obviously not suppress that effect.

\section{CONCLUDING REMARKS}

Based on a semianalytical quadridirectional eigenmode expansion technique (QUEP), a series of virtual experiments in the framework of a simple two-dimensional (2-D) PSTM model have been carried out. We adopted typical parameters for high contrast, single-mode slab waveguides in the $\mathrm{SiO}_{2} / \mathrm{Si}_{3} \mathrm{~N}_{4}$ material system. The simulations led to the following observations.

Even in cases where a purely evanescent field surrounds the sample, e.g., for a guided mode traveling along an ideal waveguide core, the photon-scanning tunneling microscopy (PSTM) signal is not strictly proportional to the local field intensity, if the dependence on the probe-sample distance is considered. Single distortions in the sample surface, or abrupt discontinuities between different homogeneous or periodic sample segments, act as localized sources that can cause pronounced direct scattering into the probe. At these probe locations, the signal exhibits strong peaks that are not present in the local intensity of the sample field. Around the peaks, interference effects can lead to an underestimation of the sample intensity by the PSTM signal. If the sample supports resonant states, the presence of the probe can cause a breakdown of an existing resonance, or the build-up of an otherwise nonexisting one. In these cases, the probe changes significantly the global optical state of the sample. Unfortunately, all these effects occur where a precise field measurement would be most interesting.

With the exception of these resonant configurations, for transverse-electric (TE)-polarized light, we found systematically only very minor changes in the local field strength at the tip position due to the presence of the probe. In the transverse-magnetic (TM) case, however, the example of the uncorrugated slab showed that the changes in the local intensity at the tip end can be much more pronounced. These observations should be of relevance for PSTM experiments that rely on the local density of states at the tip of the probe, e.g., for attempts to detect fluorescence properties of single molecules [20], [39]. For specific configurations, where a purely evanescent sample field consists of guided modes with identical profile shapes, and where the probe is kept at constant height above the sample, a reasonable recording of the optical wave patterns in the sample is possible, e.g., the observation of periods of (partially) standing waves. Despite the simplicity of the model, we found an ample qualitative agreement of these simulations with real PSTM experiments on a waveguide Bragg grating.

A number of modifications of the measurement technique can easily be modeled with the present tools. On the one hand, this concerns different schemes of illumination and detection: a localized illumination via the probe, with the transmission through the sample detected as the signal [40], and alternatively a nonlocal illumination of the sample by a focused external 
beam, with the light collected by the probe, on a side opposite to the illumination, as the signal [11]. On the other hand, different modes of signal recording could be considered: by evaluating the interference with a reference wave, phase maps of the local electromagnetic field around the sample can be generated [6], [36], [41]. In all cases, a numerical assessment of the microscopy techniques within the present model should be straightforward.

Concerning the question as to how far an experimental PSTM signal can be regarded as a quantitative "map" of the "optical field" in the device under test, we could point out several configurations where, according to the model, this simple notion definitely fails. Either the proportionality between the PSTM signal and the local intensity is entirely lost, or, even worse, the presence of the probe influences significantly the optical state of the sample. In fact, we could confirm an approximate proportionality exclusively for the constant-height scans along configurations with local single-mode standing-wave patterns. Disturbance effects must be expected, e.g., in any PSTM observations of high-quality resonances in photonic microcavities and nanocavities, in principle. While one should certainly be aware of these findings when executing PSTM measurements on photonic structures, we do not think it adequate, however, to attempt, only on the basis of the simplifying 2-D model, any more general quantitative characterization of experimental settings where these artefacts occur, or where they can be excluded.

\section{ACKNOWLEDGMENT}

The authors would like to thank E. van Groesen and H. J. W. M. Hoekstra for fruitful discussions about the numerical simulations, and E. Flück, L. Kuipers, and N. F. van Hulst for valuable information about the details of the photonscanning tunneling microscopy (PSTM) experiments. E. Flück kindly provided the experimental data for Fig. 15.

\section{REFERENCES}

[1] M. L. M. Balistreri, J. P. Korterik, G. J. Veldhuis, L. Kuipers, and N. F. van Hulst, "Quantitative photon tunneling and shear-force microscopy of planar waveguide splitters and mixers," J. Appl. Phys., vol. 89, no. 6, pp. 3307-3314, 2001.

[2] D. P. Tsai, H. E. Jackson, R. C. Reddick, S. H. Sharp, and R. J. Warmack, "Photon scanning tunneling microscope study of optical waveguides," Appl. Phys. Lett., vol. 56, no. 16, pp. 1515-1517, 1990.

[3] C. D. Poweleit, D. H. Naghski, S. M. Lindsay, J. T. Boyd, and H. E. Jackson, "Near-field scanning optical microscopy measurements of optical intensity distributions in semiconductor channel waveguides," Appl. Phys. Lett., vol. 69, no. 23, pp. 3471-3473, 1996.

[4] G. H. Vander Rhodes, B. B. Goldberg, M. S. Ünlü, S. T. Chu, W. Pan, T. Kaneko, Y. Kokobun, and B. E. Little, "Measurement of internal spatial modes and local propagation properties in optical waveguides," Appl. Phys. Lett., vol. 75, no. 16, pp. 2368-2370, 1999.

[5] M. L. M. Balistreri, J. P. Korterik, L. Kuipers, and N. F. van Hulst, "Photon scanning tunneling microscopy with a three-dimensional multiheight imaging mode," Appl. Phys. Lett., vol. 77, no. 25, pp. 4092-4094, 2000.

[6] - "Phase mapping of optical fields in integrated optical waveguide structures," J. Lightw. Technol., vol. 19, no. 8, pp. 1169-1176, Aug. 2001.

[7] - "Visualization of mode transformation in a planar waveguide splitter by near-field optical phase imaging," Appl. Phys. Lett., vol. 79, no. 7, pp. 910-912, 2001.
[8] C. Peeters, E. Flück, A. M. Otter, M. L. M. Balistreri, J. P. Korterik, L. Kuipers, and N. F. van Hulst, "Photon scanning tunneling microscopy of tailor-made photonic structures," Appl. Phys. Lett., vol. 77, no. 1, pp. $142-144,2000$.

[9] E. Flück, M. Hammer, A. M. Otter, J. P. Korterijk, L. Kuipers, and N. F. van Hulst, "Amplitude and phase evolution of optical fields inside periodic photonic structures," J. Lightw. Technol., vol. 21, no. 5, pp. 1384-1393, May 2003.

[10] A. L. Campillo, J. W. P. Hsu, C. A. White, and A. Rosenberg, "Mapping the optical intensity distribution in photonic crystals using a nearfield scanning optical microscope," J. Appl. Phys., vol. 89, no. 5, pp. 2801-2807, 2001.

[11] P. Kramper, A. Birner, M. Agio, C. M. Soukoulis, F. Müller, U. Gösele, J. Mlynek, and V. Sandoghdar, "Direct spectroscopy of a deep two-dimensional photonic crystal microresonator," Phys. Rev. B, Condens. Matter, vol. 64, p. $233102,2001$.

[12] K. Okamoto, M. Lončar, T. Yoshie, A. Scherer, Y. Qui, and P. Gogna, "Near-field scanning optical microscopy of photonic crystal nanocavities," Appl. Phys. Lett., vol. 82, no. 11, pp. 1676-1678, 2003.

[13] D. J. W. Klunder, M. L. M. Balistreri, F. C. Blom, H. J. W. M. Hoekstra, A. Driessen, L. Kuipers, and N. F. van Hulst, "High-resolution photonscanning tunneling microscope measurements of the whispering gallery modes in a cylindrical microresonator," IEEE Photon. Technol. Lett., vol. 12, no. 11, pp. 1531-1533, Nov. 2000.

[14] M. L. M. Balistreri, D. J. W. Klunder, F. C. Blom, A. Driessen, J. P. Korterik, L. Kuipers, and N. F. van Hulst, "Experimental analysis of the whispering-gallery modes in a cylindrical optical microcavity," J. Opt. Soc. Amer. B, Opt. Phys., vol. 18, no. 4, pp. 465-471, 2001.

[15] D. J. W. Klunder, M. L. M. Balistreri, F. C. Blom, H. J. W. M. Hoekstra, A. Driessen, L. Kuipers, and N. F. van Hulst, "Detailed analysis of the intracavity phenomena inside a cylindrical microresonator," J. Lightw. Technol., vol. 20, no. 3, pp. 519-529, Mar. 2002.

[16] L. Novotny, D. W. Pohl, and P. Regli, "Light propagation through nanometer-sized structures: the two-dimensional-aperture scanning near-field optical microscope," J. Opt. Soc. Amer. A, Opt. Image Sci., vol. 11, no. 6, pp. 1768-1779, 1994.

[17] _ "Near-field, far-field and imaging properties of the 2-D aperture SNOM," Ultramicroscopy, vol. 57, pp. 180-188, 1995.

[18] B. Hecht, D. W. Pohl, H. Heinzelmann, and L. Novotny, "Tunnel near-filed optical microscopy: TNOM-2," Ultramicroscopy, vol. 61, pp. 99-104, 1995.

[19] A. Castiaux, A. Dereux, J.-P. Vigneron, C. Girard, and O. J. F. Martin, "Electromagnetic fields in two-dimensional models of near-field optical microscope tips," Ultramicroscopy, vol. 60, pp. 1-9, 1995.

[20] R. X. Bian, R. C. Dunn, and X. S. Xie, "Single molecule emission characteristics in near-field microscopy," Phys. Rev. Lett., vol. 75, no. 26, pp. 4772-4775, 1995.

[21] L. Novotny, D. W. Pohl, and B. Hecht, "Scanning near-field optical probe with ultrasmall spot size," Opt. Lett., vol. 20, no. 9, pp. 970-972, 1995.

[22] L. Novotny, R. X. Brian, and X. S. Xie, "Theory of nanometric optical tweezers," Phys. Rev. Lett., vol. 79, no. 4, pp. 645-648, 1997.

[23] G. von Freymann, T. Schimmel, M. Wegener, B. Hanewinkel, A. Knorr, and S. W. Koch, "Computer simulations on near-field optical microscopy: Can subwavelength resolution be obtained using uncoated optical fiber probes?," Appl. Phys. Lett., vol. 73, no. 9, pp. 1170-1172, 1998.

[24] C. Hafner, The Generalized Multipole Technique for Computational Electromagnetics. Boston, MA: Artech House, 1990.

[25] O. J. F. Martin, A. Dereux, and C. Girard, "Iterative scheme for computing exactly the total field propagating in dielectric structures of arbitrary shape," J. Opt. Soc. Amer. A, Opt. Image Sci., vol. 11, no. 3, pp. 1073-1080, 1994.

[26] O. J. F. Martin, C. Girard, and A. Dereux, "Generalized field propagator for electromagnetic scattering and light confinement," Phys. Rev. Lett., vol. 74 , no. 4 , pp. 526-529, 1995

[27] K. S. Yee, "Numerical solution of boundary value problems involving Maxwell's equations in isotropic media," IEEE Trans. Antennas Propagat., vol. 14, no. 3, pp. 302-307, May 1966.

[28] A. Taflove, Computational Electrodynamis: The Finite Difference Time Domain Method. Norwood, MA: Artech House, 1995.

[29] S. Fan, I. Appelbaum, and J. D. Joannopoulos, "Near-field scanning optical microscopy as a simultaneous probe of fields and band structure of photonic crystals: A computational study," Appl. Phys. Lett., vol. 75, no. 22, pp. 3461-3463, 1999 .

[30] M. Hammer, "Quadridirectional eigenmode expansion scheme for 2-D modeling of wave propagation in integrated optics," Opt. Commun., vol. 235, no. 4-6, pp. 285-303, 2004. 
[31] C. Vassallo, Optical Waveguide Concepts. Amsterdam, The Netherlands: Elsevier, 1991.

[32] G. Sztefka and H. P. Nolting, "Bidirectional eigenmode propagation for large refractive index steps," IEEE Photon. Technol. Lett., vol. 5, no. 5, pp. 554-557, May 1993.

[33] J. Willems, J. Haes, and R. Baets, "The bidirectional mode expansion method for two-dimensional waveguides: the TM case," Opt. Quantum Electron., vol. 27, pp. 995-1007, 1995.

[34] M. Lohmeyer and R. Stoffer, "Integrated optical cross strip polarizer concept," Opt. Quantum Electron., vol. 33, no. 4/5, pp. 413-431, 2001.

[35] M. Lohmeyer, "Mode expansion modeling of rectangular integrated optical microresonators," Opt. Quantum Electron., vol. 34, no. 5, pp. 541-557, 2002.

[36] A. L. Campillo and J. W. P. Hsu, "Intensity and phase mapping of guided light in $\mathrm{LiNbO}_{3}$ waveguides with an interferometric near-field scanning optical microscope," Appl. Opt., vol. 42, no. 36, pp. 7149-7156, 2003.

[37] R. Stoffer, H. J. W. M. Hoekstra, R. M. de Ridder, E. van Groesen, and F. P. H. van Beckum, "Numerical studies of 2-D photonic crystals: waveguides, coupling between waveguides and filters," Opt. Quantum Electron., vol. 32, pp. 947-961, 2000.

[38] A. Suryanto, E. van Groesen, M. Hammer, and H. J. W. M. Hoekstra, "A finite element scheme to study the nonlinear optical response of a finite grating without and with defect," Opt. Quantum Electron., vol. 35, no. 4, pp. 313-332, 2003.
[39] N. F. van Hulst, J.-A. Veerman, M. F. García-Parajó, and L. Kuipers, "Analysis of individual (macro)molecules and proteins using near-field optics," J. Chem. Phys., vol. 112, no. 18, pp. 7799-7810, 2000.

[40] E. Flück, N. F. van Hulst, W. L. Vos, and L. Kuipers, "Near-field investigation of three-dimensional photonic crystals," Phys. Rev. E, Stat. Phys. Plasmas Fluids Relat. Interdiscip. Top., vol. 68, p. 015 601(R), 2003.

[41] M. L. M. Balistreri, J. P. Korterik, L. Kuipers, and N. F. van Hulst, "Local observations of phase singularities in optical fields in waveguide structures," Phys. Rev. Lett., vol. 85, no. 2, pp. 294-297, 2000.

Manfred Hammer, photograph and biography not available at the time of publication.

Remco Stoffer, photograph and biography not available at the time of publication. 